\title{
Current-driven skyrmion depinning in magnetic granular films
}

\author{
A. Salimath, ${ }^{1, *}$ A. Abbout, ${ }^{1}$ A. Brataas, ${ }^{2}$ and A. Manchon ${ }^{1, \dagger}$ \\ ${ }^{1}$ King Abdullah University of Science and Technology (KAUST), Physical Science and Engineering Division (PSE), \\ Thuwal 23955-6900, Saudi Arabia \\ ${ }^{2}$ Center for Quantum Spintronics, Department of Physics, Norwegian University of Science and Technology, NO-7491 Trondheim, Norway
}

(Received 27 August 2018; revised manuscript received 14 February 2019; published 12 March 2019)

\begin{abstract}
We consider current-driven motion of magnetic skyrmions in granular magnetic films. The study uses micromagnetic modeling and phenomenological analysis based on the Thiele formalism. Remarkably, the disorder enhances the effective skyrmion Hall effect that depends on the magnitude of the driving force (the current density and nonadiabaticity parameter). The origin is the sliding motion of the skyrmion along the grain boundaries, followed by pinning and depinning at the grain junctions. A side jump can occur during this depinning process. In addition, the critical current that triggers the skyrmion motion depends on the relative size of the crystallites with respect to the skyrmion size. Finally, when the skyrmion trajectory is confined along an edge by the nonadiabatic Magnus force, the critical current density can be significantly reduced. Our results imply that narrow nanowires exhibit higher skyrmion mobilities.
\end{abstract}

DOI: 10.1103/PhysRevB.99.104416

\section{INTRODUCTION}

Twisted spin textures called skyrmions [1-3], obtained in chiral magnets with bulk [4-9] or interfacial DzyaloshinskiiMoriya interaction (DMI) [10-14], and recently observed at room temperature [15-19], are promising candidates for the next generation digital storage and processing applications [20-22]. In addition to racetrack memory devices [23,24], skyrmions have been proposed as building blocks for transistors [25], logic applications [26-28], and neuromorphic computing [29-34]. Their rich topological properties combined with the ultralow energy required to manipulate them give them an edge over their predecessors such as domain walls and magnetic vortices [3,20,23,35-39]. Since their discovery, considerable research has been devoted to understand their topological properties, room temperature stability, and robustness against disorder [3,18-20,35-40]. Still, a deep understanding of their dynamic behavior in real thin films characterized by granular boundaries is lacking. Magnetic/nonmagnetic thin films used in technological applications are deposited using magneton sputtering techniques and, therefore, exhibit polycrystallinity, resulting in fluctuations of the magnetic properties such as magnetic anisotropy or DMI $[40,41]$. In micromagnetic simulations, the magnetic systems are typically assumed homogeneous and the effect of the material parameters variations are disregarded.

However, the most recent experiments show that disorder substantially impacts current-driven motion of magnetic solitons (domain walls and skyrmions) and cannot be neglected $[40,42,43]$. While previous studies have focused on the effect of confining potentials $[44,45]$ and random point defects [46-50], a detailed description of the skyrmion motion in polycrystalline systems remains lacking. In the present

\footnotetext{
*akshaykumar.salimath@kaust.edu.sa

†aurelien.manchon@kaust.edu.sa
}

work, we investigate current-driven motion of skyrmions in polycrystalline samples using micromagnetic modeling and phenomenological analysis. We find that the disorder enhances the effective skyrmion Hall effect that depends on the magnitude of the driving force (current density and nonadiabaticity parameter). It is the sliding motion of the skyrmion along the grain boundaries that causes this enhancement, as confirmed by simulations and Thiele's rigid motion model. In addition, our simulations show that the critical depinning current depends on the relative size of the crystallite with respect to the skyrmion size. The skyrmion motion is less influenced by the pinning forces when its motion is confined by the sample edge.

\section{SKYRMION MOTION IN POLYCRYSTALLINE MATERIALS}

\section{A. Micromagnetic simulation setup}

The micromagnetic simulations are performed using the OOMMF open source software package [51]. In the present work, the motion is induced by spin transfer torque, as described by the Landau-Lifshitz-Gilbert equation [52],

$$
\begin{aligned}
\partial_{t} \mathbf{m}= & -\gamma \mu_{0} \mathbf{m} \times \mathbf{H}_{\mathrm{eff}}+\alpha \mathbf{m} \times \partial_{t} \mathbf{m} \\
& -v_{s} \partial_{x} \mathbf{m}+\beta v_{s} \mathbf{m} \times \partial_{x} \mathbf{m},
\end{aligned}
$$

where $\mu_{0} \mathbf{H}_{\text {eff }}=-\left(1 / M_{s}\right) \delta W / \delta \mathbf{m}$ is the effective field, $W$ being the magnetic energy density and $M_{s}$ the saturation magnetization, $v_{s}=\left(J_{e} P g \mu_{B}\right) /\left(2 e M_{S}\right)$ is the driving electron velocity, $J_{e}$ is the current density applied along $\mathbf{x}, P$ is the spin polarization, and $\beta$ is the nonadiabicity parameter. Electrons flow towards the right for $v_{s}>0$. The energy density $W$ is

$$
\begin{aligned}
W= & A \sum_{i}\left(\partial_{i} \mathbf{m}\right)^{2}-K(\mathbf{n} \cdot \mathbf{m})^{2}-\frac{\mu_{0}}{2} M_{s} \mathbf{m} \cdot \mathbf{H}_{d} \\
& -\mu_{0} M_{s} \mathbf{m} \cdot \mathbf{H}-D_{\mathrm{DM}} \mathbf{m} \cdot[(\mathbf{z} \times \nabla) \times \mathbf{m}] .
\end{aligned}
$$




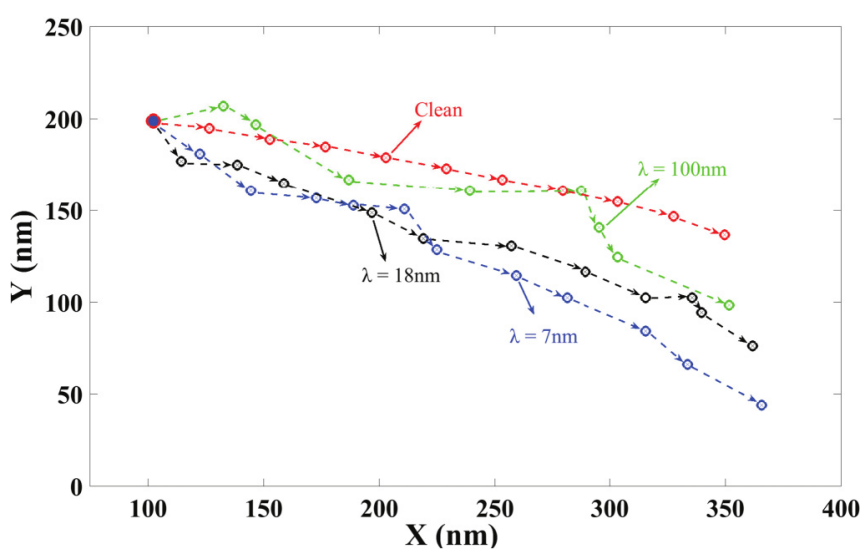

FIG. 1. Simulated skyrmion trajectory for different grain sizes. The nonadiabatic parameter $\beta=0.3<\alpha$. The other parameters are in the main text. The effective skyrmion Hall effect increases with disorder.

Here $A$ is the magnetic exchange, $K$ is the uniaxial magnetic anisotropy constant along direction $\mathbf{n}, \mathbf{H}$ is the applied magnetic field, and $\mathbf{H}_{d}$ is the demagnetization field, while $D_{\mathrm{DM}}$ is the DMI parameter.

In the simulations, we consider a ferromagnetic layer of size $400 \times 400 \times 1 \mathrm{~nm}^{3}$ with a perpendicular uniaxial anisotropy. The layer is discretized into cells of volume $2 \times 2 \times 1 / 3 \mathrm{~nm}^{3}$. The material's parameters are $M_{s}=580 \mathrm{kA} / \mathrm{m}, A=15 \mathrm{pJ} / \mathrm{m}, K=0.6 \mathrm{MJ} / \mathrm{m}^{3}$, and $D_{\mathrm{DM}}=$ $3.0 \mathrm{~mJ} / \mathrm{m}^{2}$. These parameters correspond to the experimental values for a $\mathrm{Co} / \mathrm{Pt}$ system [39]. An external magnetic field $\mu_{0} H=125 \mathrm{mT}$ is applied perpendicular to the sample plane to stabilize the skyrmion which is relaxed at a distance of $100 \mathrm{~nm}$ from the left edge of the sample. For these parameters, a relaxed skyrmion size of $18 \mathrm{~nm}$ is obtained. A large damping parameter of $\alpha=0.5$ is considered in the study, close to the experimental conditions [43]. Considering a lower value of damping coefficient does not alter our final conclusions, but only results in a shift in critical current (not shown). The disorder is modeled as crystallites with uniform magnetic anisotropy strength and varying anisotropy direction. These crystallites are generated following a random grain map obtained from Voronoi tessellation [53,54]. In this approach, the average grain size is parametrized by $\lambda$. The anisotropy axis assigned to each grain is picked randomly within the conical region symmetric about the $\mathbf{z}$ axis with a semiangle defined by $\theta$. $\theta$ defines the disorder strength. All micromagnetic simulations have been averaged over 10-25 realizations for each configuration of grain size $\lambda$ and disorder strength $\theta$. The effects of temperature are left for future work (see Sec. II D).

\section{B. Enhanced skyrmion Hall effect and sliding motion}

The skyrmion is driven by an in-plane spin polarized current along the $\mathbf{x}$ axis. The skyrmion trajectory for a specific disorder configuration and different grain sizes near the depinning threshold is shown in Fig. 1. The corresponding animations of the skyrmion motion can be found in the Supplemental Material [55]. In the clean regime, the skyrmion Hall effect is given by $\vartheta_{\mathrm{H}}=v_{y} / v_{x} \sim \alpha-\beta[56,57]$ (see dis-

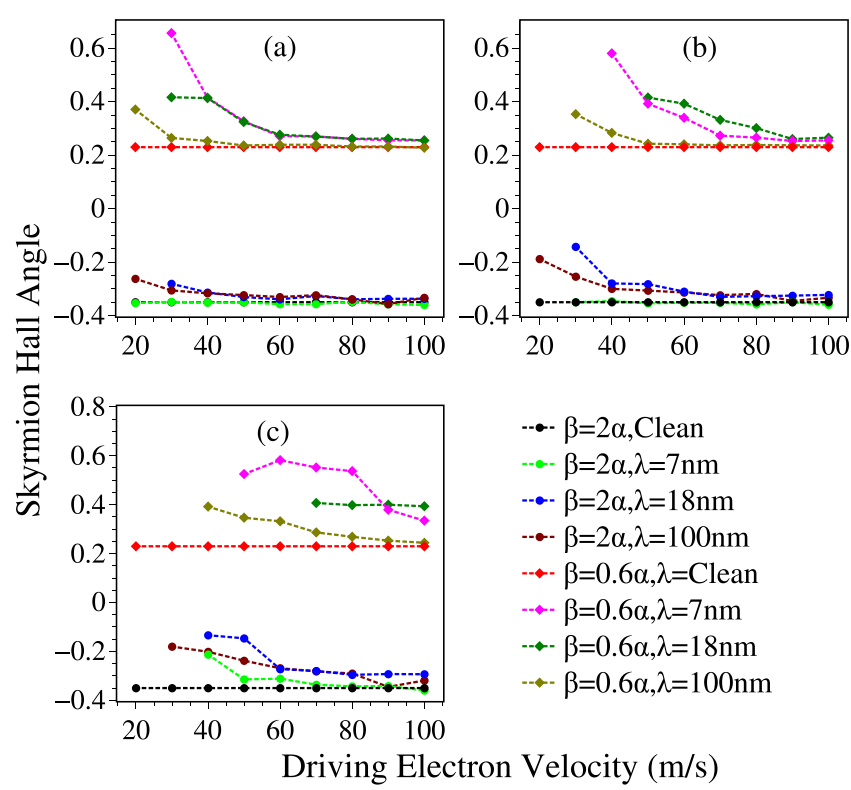

FIG. 2. Simulated skyrmion Hall angle as a function of current density for several grain sizes, nonadiabaticity parameters, and disorder strengths. (a) $\theta=2^{0}$, (b) $\theta=3^{0}$, (c) $\theta=5^{0}$.

cussion below). However, in the presence of polycrystallinity the skyrmion Hall effect is enhanced, an effect that is prominent in the case of small crystallites and reduced in the case of large crystallites.

A detailed study of the skyrmion Hall angle as a function of the driving electron velocity $v_{s}$ is shown in Fig. 2 for various disorder strengths [(a) $\theta=2^{0}$, (b) $\theta=3^{0}$, (c) $\theta=5^{0}$, and grain sizes $(\lambda=7,18,100$, and $+\infty \mathrm{nm})$. For completeness, the simulations were performed for two different nonadiabaticity parameters, $\beta=0.6 \alpha$ and $\beta=2 \alpha$, corresponding to positive and negative skyrmion Hall effect, respectively. In a clean system, the skyrmion Hall angle is independent of the drive current for given $\alpha$ and $\beta$ parameters [56]. However, crystallites enhance the skyrmion Hall angle substantially, particularly close to the current threshold. Furthermore, the smaller the grains, the larger the skyrmion Hall angle, in agreement with Fig. 1. Interestingly, the skyrmion Hall angle is significantly reduced upon increasing the driving current density, such that in the limit of large driving currents, the skyrmion Hall effect converges towards the results obtained in the clean limit, irrespective of the strength of disorder or the grain size.

To better understand the enhanced skyrmion Hall effect, we investigate the skyrmion trajectory in a polycrystalline landscape. Figures 3(a)-3(c) display the simulated skyrmion trajectory in a sample with an average grain size of $57 \mathrm{~nm}$ for different driving current densities. The animations of the simulations are available in the Supplemental Material [55]. At low current densities [Fig. 3(a)], the driving force is insufficient to overcome the repulsive potential formed by the grain boundaries. Consequently, the skyrmion exhibits a sliding motion along the boundary. This motion channels the skyrmion towards the grain junction where it gets pinned. For intermediate current density [Fig. 3(b)], the skyrmion passes the first grain boundary and slides on the second grain 



FIG. 3. Skyrmion motion in a polycrystalline sample. (a)-(c) Skyrmion trajectory in a sample with average grain size $57 \mathrm{~nm}$, disorder strength $\theta=5^{0}$, and for increasing driving current densities. (d)-(f) Skyrmion trajectory in a sample with average grain size $57 \mathrm{~nm}$, disorder strength $\theta=5^{\circ}$, and several realizations of anisotropy axis direction for increasing driving current densities. The color scale represents the azimuthal angle of the magnetic moment, as shown in (a). (g) Skyrmion Hall angle as a function of current density for disorder strength $\theta=5^{0}$ and for several grain sizes and several realizations of anisotropy axis direction.

boundary before getting depinned. By further increasing the current density [Fig. 3(c)], the skyrmion becomes less sensitive to the grain boundaries and therefore exhibits a reduced sliding motion. As a result, the overall trajectory of the skyrmion becomes more straight upon increasing the current density, thereby reducing the overall skyrmion Hall effect.

In order to test the reproducibility of this behavior, we performed additional simulations on different randomness realizations for a given grain configuration. The results are reported in Figs. 3(d)-3(f) for six different anisotropy disorder configurations. The other parameters (grain configuration, disorder strength, and current densities) are the same as in Figs. 3(a)-3(c). The skyrmion behavior near the depinning threshold is determined by the grain boundary orientation, resulting in various trajectories depending on the specific disorder configuration. Nevertheless, the basic concept of sliding motion along the grain boundary followed by pinning/depinning at the junctions remains valid. At high current densities though, the trajectory is much less sensitive to the specific disorder configuration and is determined by the driving current density and the nonadiabicity parameter. In Fig. 3(g), we show the Hall angle as a function of the applied current density, averaged over 25 different anisotropy disorder configurations and for various grain sizes. As expected, the Hall angle decreases upon increasing the depinning current density, confirming the effect obtained in Fig. 2.

The sliding motion can be accounted for by using Thiele's equation for rigid motion [56-58],

$$
\mathbf{G} \times\left(\mathbf{v}_{s}-\mathbf{v}_{d}\right)+\mathcal{D}\left(\beta \mathbf{v}_{s}-\alpha \mathbf{v}_{d}\right)+\mathbf{F}_{\text {pin }}=0,
$$

where $\mathbf{G}=\mathcal{G} \mathbf{z}$ is the gyromagnetic coupling vector $(\mathcal{G}=$ $4 \pi \mathcal{Q}, \mathcal{Q}$ being the skyrmion charge), $\mathcal{D}$ is the dissipative tensor (reduced to a scalar by symmetry), and $\mathbf{v}_{d}$ is the drift velocity of the skyrmion. Here $\mathbf{F}_{\text {pin }}=-\nabla V_{\text {pin }}$ is the pinning force emerging between two adjacent grains, and $V_{\text {pin }}$ is the pinning potential at the grain boundary. The pinning force is normal to the boundary and can depend on both space and the skyrmion velocity [56]. Rearranging the terms we obtain an expression for the skyrmion drift velocity,

$$
\begin{aligned}
\mathbf{v}_{d}= & \frac{\mathcal{G}^{2}+\alpha \beta \mathcal{D}^{2}}{\mathcal{G}^{2}+\alpha^{2} \mathcal{D}^{2}} \mathbf{v}_{s}+(\alpha-\beta) \frac{\mathcal{D} \mathcal{G}}{\mathcal{G}^{2}+\alpha^{2} \mathcal{D}^{2}}\left(\mathbf{z} \times \mathbf{v}_{s}\right) \\
& +\alpha \frac{\mathcal{D}}{\mathcal{G}^{2}+\alpha^{2} \mathcal{D}^{2}} \mathbf{F}_{\text {pin }}-\frac{\mathcal{G}}{\mathcal{G}^{2}+\alpha^{2} \mathcal{D}^{2}} \mathbf{z} \times \mathbf{F}_{\text {pin }} .
\end{aligned}
$$

The first and the second terms in Eq. (4) account for the skyrmion longitudinal and transverse velocity components in the disorder-free regime, respectively. The third term is the contribution of the repulsive force from the pinning center and the fourth term corresponds to the gyrotropic motion normal to the direction of pinning force. In the absence of a pinning force, the skyrmion Hall angle is $\vartheta_{\mathrm{H}}=v_{d}^{y} / v_{d}^{x}=$ $(\alpha-\beta) \mathcal{D} G /\left(\mathcal{G}^{2}+\alpha^{2} \mathcal{D}^{2}\right)$, as mentioned above. However, in the presence of disorder, since the pinning force $\mathbf{F}_{\text {pin }}$ depends on spatial coordinates [47] (and possibly on the drift velocity $\mathbf{v}_{d}$ [56]), Eq. (4) is not a closed-form solution and needs to be solved self-consistently.

To illustrate the influence of the pinning force on the skyrmion motion, let us consider the case depicted in Fig. 4(a), where a magnetic skyrmion driven by a current along the direction $\mathbf{v}_{s}$ impinges on a grain boundary that forms an angle of $\phi$ with $\mathbf{v}_{s}$. We parse the skyrmion drift velocity into

$$
\mathbf{v}_{d}=v_{d}^{\|} \mathbf{e}_{\|}+v_{d}^{\perp} \mathbf{e}_{\perp},
$$

where $\mathbf{e}_{\|(\perp)}$ is a unit vector along (normal to) the boundary, as indicated in Fig. 4(a). In addition, the pinning force is $\mathbf{F}_{\text {pin }}=$ $F_{\text {pin }}\left(\mathbf{v}_{d}\right) \mathbf{e}_{\perp}$. At low current densities, we can impose $v_{d}^{\perp}=0$ and deduce the sliding velocity along the boundary,

$$
v_{d}^{\|}=\frac{\mathcal{G} \sin \phi+\beta \mathcal{D} \cos \phi}{\alpha \mathcal{D}} v_{s} .
$$

The condition for which the skyrmion crosses the boundary reads $v_{d}^{\perp} \leqslant 0$. Therefore, the critical driving current density needed to expel the skyrmion from the grain boundary is

$$
v_{s}^{c}=\frac{\alpha \mathcal{D}}{\left(\mathcal{G}^{2}+\alpha \beta \mathcal{D}^{2}\right) \sin \phi-(\alpha-\beta) \mathcal{D} G \cos \phi} F_{\text {pin }}^{c},
$$



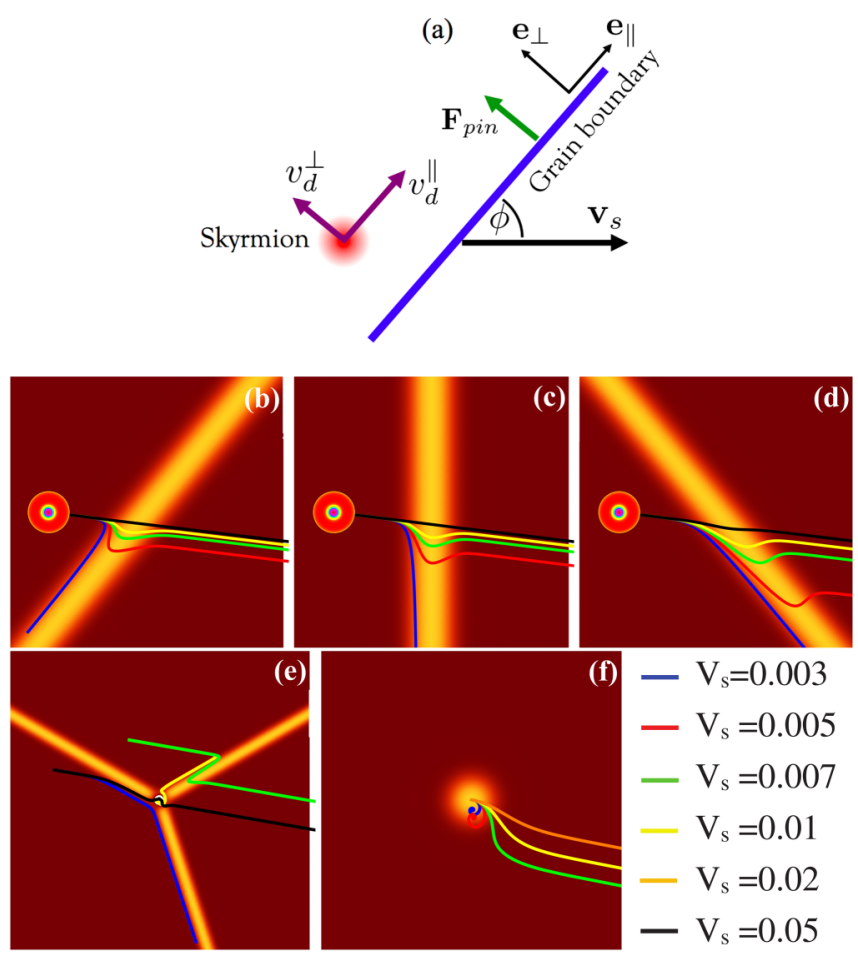

$-\mathrm{V}_{\mathrm{s}}=0.005$

$-\mathrm{V}_{\mathrm{s}}=0.007$

$-\mathrm{V}_{\mathrm{s}}=0.01$

- $\mathrm{V}_{\mathrm{s}}=0.02$

$-\mathrm{V}_{\mathrm{s}}=0.05$

FIG. 4. Skyrmion trajectory along a grain boundary obtained from Thiele's equation. (a) Schematics of skyrmion motion along a grain boundary. (b)-(e) Skyrmion trajectories obtained by numerically solving Eq. (4) with a Gaussian pinning potential. These calculations describe the skyrmion sliding behavior along different grain boundary orientations. (f) Skyrmion trajectory upon depinning from local defect. We have considered $\beta=0.3$ and assumed $\mathcal{G}=\mathcal{D}$.

where $F_{\text {pin }}^{c}=F_{\text {pin }}\left(\mathbf{v}_{d}^{c}\right)$ is the pinning force at the depinning threshold. Hence, the critical skyrmion sliding velocity before it is expelled from the repulsive potential reads

$$
v_{d}^{\|, c}=\frac{\mathcal{G} \sin \phi+\beta \mathcal{D} \cos \phi}{\left(\mathcal{G}^{2}+\alpha \beta \mathcal{D}^{2}\right) \sin \phi-(\alpha-\beta) \mathcal{D} G \cos \phi} F_{\text {pin }}^{c} .
$$

These phenomenological expressions reveal two important features: Both the sliding velocity, Eq. (6), and the critical depinning current, Eq. (7), depend on the orientation of the grain boundary thereby contributing to a "side jump" of the skyrmion.

These effects are further revealed in Figs. 4(b)-4(d) which display the skyrmion velocity when approaching a grain boundary for various boundary orientations. These results are obtained by numerically solving Eq. (4) while assuming a quasi-one-dimensional repulsive Gaussian pinning potential of the form $V_{\text {pin }}(x, y)=V_{0} e^{-\frac{(x \sin \phi-y \cos \phi)^{2}}{R^{2}}}$, where $\phi$ is the angle of the boundary. Here $V_{0}$ is the strength of the interaction and $R$ determines the interaction range. In agreement with our micromagnetic simulations and with the analytical expressions provided above, at low driving current (blue curve) the driving force cannot overcome the potential of the grain boundary, such that the skyrmion remains "stuck" along the boundary, exhibiting the sliding motion discussed above. Upon increasing the driving current, the skyrmion can escape from the grain boundary after a short sliding regime, thereby reducing the effective Hall angle.
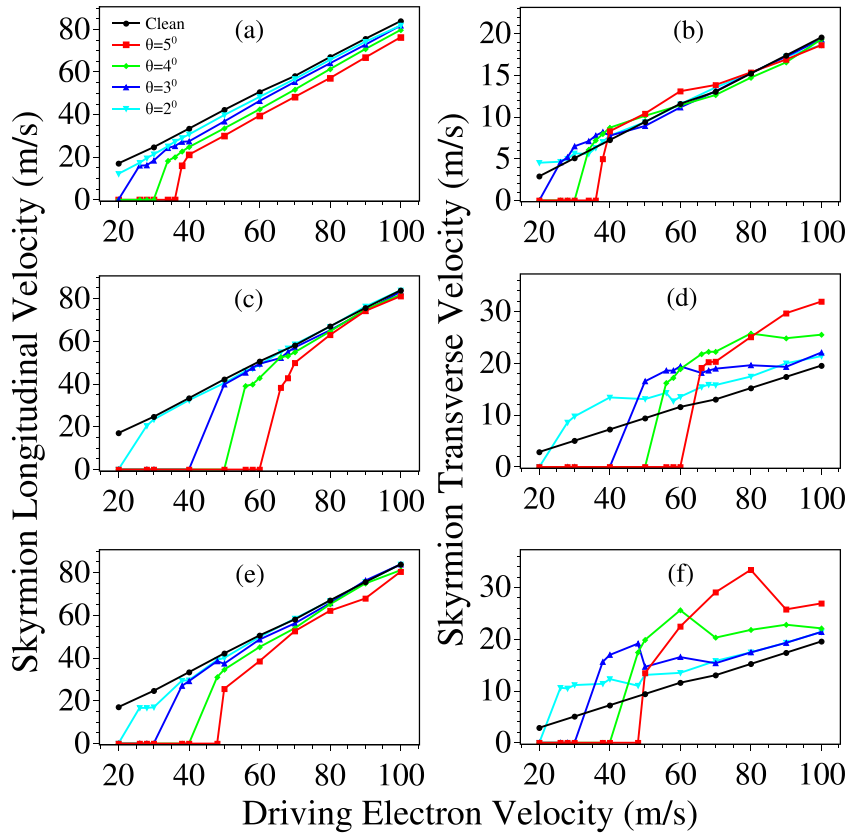

FIG. 5. Skyrmion longitudinal and transverse velocity components as a function of the driving current for an average grain size of (a) and (b) $\lambda=100 \mathrm{~nm}$, (c) and (d) $\lambda=18 \mathrm{~nm}$, and (e) and (f) $\lambda=7 \mathrm{~nm}$. Here $\beta=0.3$ and the other parameters are given in the main text.

Interestingly, the distance over which the sliding occurs depends on the angle between the driving current direction and the boundary. This distance is larger for $\phi<0$ [Fig. 4(d)] than for $\phi>0$ [Fig. 4(b)]. In the former case the skyrmion slides along the direction of the driving current, while in the latter case it slides against it. This skewness is directly attributed to the influence of the gyrotropic force $-\mathcal{G}$. Notice also that the sliding distance reduces upon increasing the driving current amplitude, as observed in the micromagnetic simulations in Fig. 3.

\section{Skyrmion depinning}

In the previous section, we highlighted that skyrmion sliding along grain boundaries has the tendency of channeling the skyrmion towards grain junctions, as illustrated in Fig. 4(e). Close to such junctions, the skyrmion has a high probability of getting pinned, a probability that decreases upon increasing the driving current density. Let us now investigate the skyrmion depinning behavior (at zero temperature) in polycrystalline samples.

Figures 5(a)-5(f) displays the simulated longitudinal and transverse skyrmion velocity components as a function of the driving current density for different grain sizes and disorder strengths. The simulations are performed for the case when $\alpha>\beta$. Depending on the grain size and the disorder strength, we observed significant pinning in these samples. The disorder suppresses the longitudinal velocity component and gives rise to a threshold driving current density, the value of which depends on the grain size, the defect density and the disorder strength [see Figs. 6(a) and 6(b)]. The critical driving current reaches a maximum when the grain size is set comparable to 

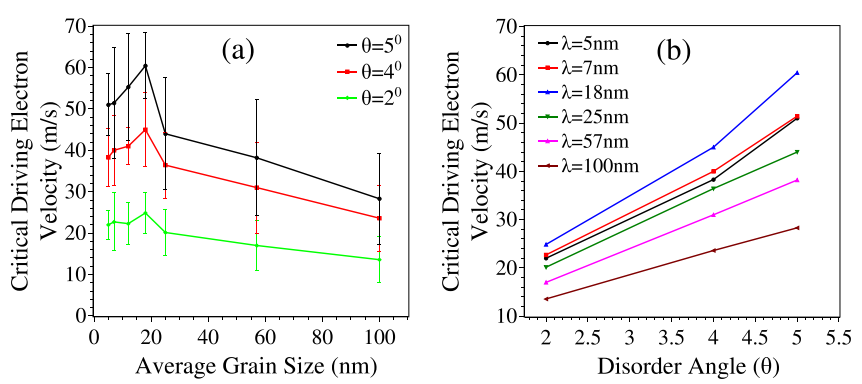

FIG. 6. (a) Critical current density as a function of average grain size. (b) Critical current density as a function of disorder strength defined by $\theta$. Here $\beta=0.3$ and the other parameters are given in the main text.

the skyrmion size, as displayed in Fig. 6(a). This is because the skyrmion then feels the complete impact of each grain. When the grain size is either reduced or enhanced, the critical driving current decreases, an effect also reported in Ref. [40]. In addition, we also observe an enhancement of the skyrmion Hall effect right after the skyrmion gets depinned [Figs. 5(b), 5(d) and 5(f)]. This "overshoot" increases upon reducing the grain size and increasing the disorder strength, and decreases upon increasing the driving current density.

By analyzing the skyrmion sliding distance and velocity using Thiele's rigid motion model, we can explain the skyrmion trajectory when the average grain size is much larger than the skyrmion size. Since the density of grain junctions is low in this case, the skyrmion speeds up along the grain boundary and most likely leaves it before it reaches the junction, as illustrated in Fig. 4(e). Because of this behavior, the skyrmion has a low probability to get pinned, resulting in a near disorder-free trajectory. At low current density though, the driving force is not large enough to overcome the boundary potential and the skyrmion is likely to be channeled towards the grain junction, leading to enhanced pinning probability.

Notice that the enhanced skyrmion Hall effect also occurs during the depinning process itself. If one considers a skyrmion originally pinned at a grain boundary or at a local defect, the Hall effect substantially depends on the velocity at which the skyrmion escapes the pinning center. This effect is illustrated in Fig. 4(f) with Thiele's model. We consider a localized pinning potential with cylindrical symmetry and compute the trajectory of the rigid skyrmion as a function of the driving current. Above the threshold, an effect similar to the one reported in Fig. 5 is observed: For a driving current close to the threshold, a "side jump" is observed, which is reduced upon increasing the current.

To complete our discussion, let us turn our attention to the influence of the nonadiabaticity parameter on the critical driving velocity. Indeed, while $\beta$ governs the longitudinal motion of skyrmions only at higher order $\left[v_{d}^{x}=\left(\mathcal{G}^{2}+\right.\right.$ $\left.\alpha \beta \mathcal{D}^{2}\right) /\left(\mathcal{G}^{2}+\alpha^{2} \mathcal{D}^{2}\right)$ ], it drives the transverse velocity when $\beta \neq \alpha[20,44,45]$. Hence, in a disordered regime dominated by sliding motion along the grain boundaries, increasing $\beta$ drives the skyrmion out of sliding motion and prevents it from being driven into a grain junction resulting in a reduced pinning, as reported in Fig. 7(a).
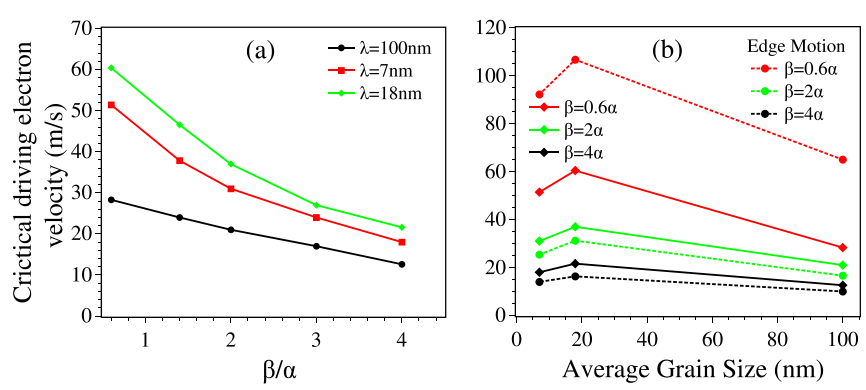

FIG. 7. (a) Simulated critical current density as a function of nonadiabaticity parameter $\beta$ for various grain sizes and a disorder strength $\theta=5^{0}$. (b) Simulated critical current density as a function of average grain size for various $\beta$ parameter. Solid (dashed) lines correspond to a motion taking place near the center (the edge) of the wire.

The competition between the Magnus force due to the nonadiabaticity parameter and the sliding motion on grain boundaries is best illustrated by considering the skyrmion depinning at the edge of a magnetic wire. There, the Magnus force due to the nonadiabaticity parameter is balanced by the confinement force. Figure 7(b) displays the critical current density as a function of the grain size for skyrmions initially located at the center (solid lines) and at the edges of the sample (dashed lines). When $\alpha<\beta$ (green and black symbols), the skyrmion is pushed towards the edge and slides along it with velocity $v_{d}^{x}=(\beta / \alpha) v_{s}[42,44,45]$, resulting in a reduced critical driving current density compared to the case of central motion. However, when $\alpha>\beta$ the skyrmion is pushed along the opposite edge of the sample, such that the influence of the confinement force is reduced. As a result, the skyrmion is more sensitive to defects and grain boundaries, leading to higher threshold current density. These observations suggest that narrow nanowires have the potential to enable high skyrmion mobility, not only by quenching the nonadiabatic Magnus force, but also by reducing the sensitivity of skyrmions to pinning.

\section{Impact of temperature}

We emphasize that the effects discussed above do not account for the impact of thermal activation. Brownian motion of skyrmions has been studied numerically [59-63] and observed experimentally $[33,34,64]$. The experiments reported skyrmion diffusion constants of $\sim \mu \mathrm{m}^{2} / \mathrm{s}$ at room temperature [33,34], which opens appealing perspectives for probabilistic computing. In addition, mechanisms for thermally induced skyrmion annihilation in clean ferromagnets have been investigated by various groups $[61,65]$. In particular, the thermal stability of skyrmions in nanotracks has been scrutinized and it has been shown that the thermal activation energy is substantially reduced at the boundary of the track compared to the one inside the track. As a result, while thermal fluctuations reduce the overall skyrmion stability, it is even more dramatic at boundaries due in part to the canting of the magnetic moments [66-68]. The same applies to nonmagnetic impurities $[69,70]$, and therefore, one can speculate that thermal fluctuations reduce the skyrmion stability at the grain boundaries. Finally, thermal fluctuations can also weaken the 
skyrmion-grain boundary interaction, which is expected to favor a low critical current density [45].

\section{CONCLUSION}

We have investigated the role of granular boundaries on the skyrmion motion in polycrystalline samples through micromagnetic simulations and Thiele formalism. We found that the critical current density depends on the relative size between the grains and the skyrmion and reaches a maximum when both are of the same order. More intriguingly, we observe that the skyrmion Hall effect is effectively enhanced by the presence of disorder. This is caused by the onset of sliding motion of the skyrmion along the grain boundaries, and side jump from grain junctions. Such an effect is overall quenched upon increasing the driving current density. Finally, we have shown that when the skyrmion trajectory is confined along an edge by the nonadiabatic Magnus force, the critical current density can be significantly reduced, suggesting that narrow nanowires yield higher skyrmion mobility.

\section{ACKNOWLEDGMENTS}

A.S., A.A., and A.M. acknowledge support from the King Abdullah University of Science and Technology (KAUST), as well as computing time and team assistance on the supercomputers SHAHEEN at KAUST Supercomputing Center. A.B. is supported by the Research Council of Norway through its Centre of Excellence funding scheme, Project No. 262633, "QuSpin."
[1] T. H. R. Skyrme, A unified field theory of mesons and baryons, Nuc. Phys. 31, 556 (1962).

[2] A. N. Bogdanov and U. K. Rößler, Chiral Symmetry Breaking in Magnetic Thin Films and Multilayers, Phys. Rev. Lett. 87, 037203 (2001).

[3] N. Nagaosa and Y. Tokura, Topological properties and dynamics of magnetic skyrmions, Nat. Nanotech. 8, 899 (2013).

[4] U. K. Rößler, A. N. Bogdanov, and C. Pfleiderer, Spontaneous skyrmion ground states in magnetic metals, Nature (London) 442, 797 (2006).

[5] S. Mühlbauer, B. Binz, F. Jonietz, C. Pfleiderer, A. Rosch, A. Neubauer, R. Georgii, and P. Böni, Skyrmion lattice in a chiral magnet, Science 323, 915 (2009).

[6] C. Pfleiderer, T. Adams, A. Bauer, W. Biberacher, B. Binz, F. Birkelbach, P. Böni, C. Franz, R. Georgii, and M. Janoschek, Skyrmion lattices in metallic and semiconducting B20 transition metal compounds, J. Phys.: Condens. Matter 22, 164207 (2010).

[7] X. Z. Yu, Y. Onose, N. Kanazawa, J. H. Park, J. H. Han, Y. Matsui, N. Nagaosa, and Y. Tokura, Real-space observation of a two-dimensional skyrmion crystal, Nature (London) 465, 901 (2010).

[8] X. Z. Yu, N. Kanazawa, Y. Onose, K. Kimoto, W. Z. Zhang, S. Ishiwata, Y. Matsui, and Y. Tokura, Near room-temperature formation of a skyrmion crystal in thin-films of the helimagnet FeGe, Nat. Mater. 10, 106 (2011).

[9] S. Seki, X. Z. Yu, S. Ishiwata, and Y. Tokura, Observation of skyrmions in a multiferroic material, Science 336, 198 (2012).

[10] S. Heinze, K. V. Bergmann, M. Menzel, J. Brede, A. Kubetzka, R. Wiesendanger, G. Bihlmayer, and S. Blügel, Spontaneous atomic-scale magnetic skyrmion lattice in two dimensions, Nat. Phys. 7, 713 (2011).

[11] N. Romming, C. Hanneken, M. Menzel, J. E. Bickel, B. Wolter, K. V. Bergmann, A. Kubetzka, and R. Wiesendanger, Writing and deleting single magnetic skyrmions, Science 341, 636 (2013).

[12] B. Dupé, M. Hoffmann, C. Paillard, and S. Heinze, Tailoring magnetic skyrmions in ultra-thin transition metal films, Nat. Commun. 5, 4030 (2014).

[13] B. Dupé, G. Bihlmayer, M. Böttcher, S. Blügel, and S. Heinze, Engineering skyrmions in transition-metal multilayers for spintronics, Nat. Commun. 7, 11779 (2016).
[14] A. Belabbes, G. Bihlmayer, F. Bechstedt, S. Blügel, and A. Manchon, Hund's Rule-Driven Dzyaloshinskii-Moriya Interaction at 3d-5d Interfaces, Phys. Rev. Lett. 117, 247202 (2016).

[15] W. Jiang, P. Upadhyaya, W. Zhang, G. Yu, M. B. Jungfleisch, F. Y. Fradin, J. E. Pearson, Y. Tserkovnyak, K. L. Wang, O. Heinonen, S. G. E. te Velthuis, and A. Hoffmann, Blowing magnetic skyrmion bubbles, Science 349, 283 (2015).

[16] G. Chen, A. Mascaraque, A. T. N'Diaye, and A. K. Schmid, Room temperature skyrmion ground state stabilized through interlayer exchange coupling, Appl. Phys. Lett. 106, 242404 (2015).

[17] C. Moreau-Luchaire, C. Moutafis, N. Reyren, J. Sampaio, C. A. F. Vaz, N. Van Horne, K. Bouzehouane, K. Garcia, C. Deranlot, P. Warnicke, P. Wohlhüter, J.-M. George, M. Weigand, J. Raabe, V. Cros, and A. Fert, Additive interfacial chiral interaction in multilayers for stabilization of small individual skyrmions at room temperature, Nat. Nanotech. 11, 444 (2016).

[18] S. Woo, K. Litzius, B. Krüger, M.- Young Im, L. Caretta, K. Richter, M. Mann, A. Krone, R. M. Reeve, M. Weigand, P. Agrawal, I. Lemesh, M.-A. Mawass, P. Fischer, M. Kläui, and G. S. D. Beach, Observation of room-temperature magnetic skyrmions and their current-driven dynamics in ultrathin metallic ferromagnets, Nat. Mater. 15, 501 (2016).

[19] O. Boulle, J. Vogel, H. Yang, S. Pizzini, D. de Souza Chaves, A. Locatelli, T. O. Menteş, A. Sala, L. D. Buda-Prejbeanu, O. Klein, M. Belmeguenai, Y. Roussigné, A. Stashkevich, S. M. Chérif, L. Aballe, M. Foerster, M. Chshiev, S. Auffret, I. M. Miron, and G. Gaudin, Room-temperature chiral magnetic skyrmions in ultrathin magnetic nanostructures, Nat. Nanotech. 11, 449 (2016).

[20] A. Fert, V. Cros, and J. Sampaio, Skyrmions on the track, Nat. Nanotech. 8, 152 (2013).

[21] A. Rosch, Spintronics: Electric control of skyrmions, Nat. Nanotech. 12, 103 (2017).

[22] W. Kang, Y. Huang, X. Zhang, Y. Zhou, and W. Zhao, Skyrmion-Electronics: An Overview and Outlook, Proc. IEEE 104, 2040 (2016).

[23] J. Müller, Magnetic skyrmions on a two-lane racetrack, New J. Phys. 19, 025002 (2017).

[24] D. Zhu, W. Kang, S. Li, Y. Huang, X. Zhang, Y. Zhou, and W. Zhao, Skyrmion racetrack memory with random information 
update/deletion/insertion, IEEE Trans. Electron Devices 65, 87 (2017).

[25] X. Zhang, Y. Zhou, M. Ezawa, G. P. Zhao, and W. Zhao, Magnetic skyrmion transistor: skyrmion motion in a voltagegated nanotrack, Sci. Rep. 5, 11369 (2015).

[26] S. Luo, M. Song, X. Li, Y. Zhang, J. Hong, X. Yang, X. Zou, N. Xu, and L. You, Reconfigurable skyrmion logic gates, Nano Lett. 18, 1180 (2018).

[27] X. Zhang, M. Ezawa, and Y. Zhou, Magnetic skyrmion logic gates: conversion, duplication and merging of skyrmions, Sci. Rep. 5, 9400 (2015).

[28] M. G. Mankalale, Z. Zhao, J.-P. Wang, and S. S. Sapatnekar, SkyLogic - A proposal for a skyrmion logic device, arXiv:1811.2016.

[29] Y. Huang, W. Kang, X. Zhang, Y. Zhou, and W. Zhao, Magnetic skyrmion-based synaptic devices, Nanotechnology 28, 08LT02 (2017).

[30] S. Li, W. Kang, Y. Huang, X. Zhang, Y. Zhou, and W. Zhao, Magnetic skyrmion-based artificial neuron device, Nanotechnology 28, 31LT01 (2017).

[31] M.-C. Chen, A. Sengupta, and K. Roy, Magnetic skyrmion as a spintronic deep learning spiking neuron processor, IEEE Trans. Magn. 54, 1500207 (2018).

[32] D. Pinna, F. Abreu Araujo, J.-V. Kim, V. Cros, D. Querlioz, P. Bessiere, J. Droulez, and J. Grollier, Skyrmion Gas Manipulation for Probabilistic Computing, Phys. Rev. Appl. 9, 064018 (2018).

[33] T. Nozaki, Y. Jibiki, M. Goto, E. Tamura, T. Nozaki, H. Kubota, A. Fukushima, S. Yuasa, and Y. Suzuki, Brownian motion of skyrmion bubbles and its control by voltage applications, Appl. Phys. Lett. 114, 012402 (2019).

[34] J. Zázvorka, F. Jakobs, D. Heinze, N. Keil, S. Kromin, S. Jaiswal, K. Litzius, G. Jakob, P. Virnau, D. Pinna, K. EverschorSitte, A. Donges, U. Nowak, and M. Kläui, Thermal skyrmion diffusion applied in probabilistic computing, arXiv:1805.5924.

[35] T. Schulz, R. Ritz, A. Bauer, M. Halder, M. Wagner, C. Franz, C. Pfleiderer, K. Everschor, M. Garst, and A. Rosch, Emergent electrodynamics of skyrmions in a chiral magnet, Nat. Phys. 8, 301 (2012).

[36] N. Nagaosa and Y. Tokura, Emergent electromagnetism in solids, Phys. Scr. T 146, 014020 (2012).

[37] A. Bisig, C. A. Akosa, J.-H. Moon, J. Rhensius, C. Moutafis, A. von Bieren, J. Heidler, G. Kiliani, M. Kammerer, M. Curcic, M. Weigand, T. Tyliszczak, B. Van Waeyenberge, H. Stoll, G. Schütz, K.-J. Lee, A. Manchon, and M. Kläui, Enhanced NonAdiabaticity in Vortex Cores Due to the Emergent Hall Effect, Phys. Rev. Lett. 117, 277203 (2016).

[38] C. A. Akosa, P. B. Ndiaye, and A. Manchon, Intrinsic nonadiabatic topological torque in magnetic skyrmions and vortices, Phys. Rev. B 95, 054434 (2017).

[39] J. Sampaio, S. Rohart, A. Thiaville, and A. Fert, Nucleation, stability and current-induced motion of isolated magnetic skyrmions in nanostructures, Nat. Nanotech. 8, 839 (2013).

[40] W. Legrand, D. Maccariello, N. Reyren, K. Garcia, C. Moutafis, C. Moreau-Luchaire, S. Collin, K. Bouzehouane, V. Cros, and A. Fert, Room-temperature current-induced generation and motion of sub-100 nm skyrmions, Nano Lett. 17, 2703 (2017).

[41] T. Matsumoto, Y.-G. So, Y. Kohno, H. Sawada, R. Ishikawa, Y. Ikuhara, and N. Shibata, Jointed magnetic skyrmion lattices at a small-angle grain boundary directly visualized by advanced electron microscopy, Sci. Rep. 6, 35880 (2016).

[42] W. Jiang, X. Zhang, G. Yu, W. Zhang, X. Wang, M. B. Jungfleisch, J. E. Pearson, X. Cheng, O. Heinonen, K. L. Wang, Y. Zhou, A. Hoffmann, and S. G. E. te Velthuis, Direct observation of the skyrmion Hall effect, Nat. Phys. 13, 162 (2017).

[43] K. Litzius, I. Lemesh, B. Krüger, P. Bassirian, L. Caretta, K. Richter, F. Büttner, K. Sato, O. A. Tretiakov, J. Förster, R. M. Reeve, M. Weigand, I. Bykova, H. Stoll, G. Schütz, G. S. D. Beach, and M. Kläui, Skyrmion Hall effect revealed by direct time-resolved X-ray microscopy, Nat. Phys. 13, 170 (2017).

[44] J. Iwasaki, M. Mochizuki, and N. Nagaosa, Current-induced skyrmion dynamics in constricted geometries, Nat. Nanotech. 8, 742 (2013).

[45] J. Iwasaki, W. Koshibae, and N. Nagaosa, Colossal spin transfer torque effect on skyrmion along the edge, Nano Lett. 14, 4432 (2014).

[46] J. Müller and A. Rosch, Capturing of a magnetic skyrmion with a hole, Phys. Rev. B 91, 054410 (2015).

[47] S.-Z. Lin, C. Reichhardt, C. D. Batista, and A. Saxena, Particle model for skyrmions in metallic chiral magnets: dynamics, pinning, and creep, Phys. Rev. B 87, 214419 (2013).

[48] C. Reichhardt, D. Ray, and C. J. O. Reichhardt, Collective Transport Properties of Driven Skyrmions with Random Disorder, Phys. Rev. Lett. 114, 217202 (2015).

[49] C. Reichhardt and C. J. O. Reichhardt, Noise fluctuations and drive dependence of the skyrmion Hall effect in disordered systems, New J. Phys. 18, 095005 (2016).

[50] S. Woo, K. M. Song, X. Zhang, Y. Zhou, M. Ezawa, X. Liu, S. Finizio, J. Raabe, N. J. Lee, S.-I. Kim, S.-Y. Park, Y. Kim, J.-Y. Kim, D. Lee, O. Lee, J. W. Choi, B.-C. Min, H. C. Koo, and J. Chang, Current-driven dynamics and inhibition of the skyrmion Hall effect of ferrimagnetic skyrmions in GdFeCo films, Nat. Commun. 9, 959 (2018).

[51] https://math.nist.gov/oommf/.

[52] M. Najafi, B. Krüger, S. Bohlens, M. Franchin, H. Fangohr, A. Vanhaverbeke, R. Allenspach, M. Bolte, U. Merkt, D. Pfannkuche, D. P. F. Möller, and G. Meier, Proposal for a standard problem for micromagnetic simulations including spintransfer torque, J. Appl. Phys. 105, 113914 (2009).

[53] J. W. Lau, R. D. McMichael, and M. J. Donahue, Implementation of two-dimensional polycrystalline grains in object oriented micromagnetic framework, J. Res. Natl Inst. Stand. Technol. 114, 57 (2009).

[54] T. Herranen and L. Laurson, Domain walls within domain walls in wide ferromagnetic strips, Phys. Rev. B 92, 100405(R) (2015).

[55] See Supplemental Material at http://link.aps.org/supplemental/ 10.1103/PhysRevB.99.104416 for movies of the dynamics of a skyrmion in a polycrystalline sample showing the sliding motion along grain boundaries and the reduction of the skyrmion Hall effect upon increasing the current magnitude.

[56] J. Iwasaki, M. Mochizuki, and N. Nagaosa, Universal currentvelocity relation of skyrmion motion in chiral magnets, Nat. Commun. 4, 1463 (2013).

[57] J. He, Z. Li, and S. Zhang, Current-driven vortex domain wall dynamics by micromagnetic simulations, Phys. Rev. B 73, 184408 (2006). 
[58] A. A. Thiele, Steady-State Motion of Magnetic Domains, Phys. Rev. Lett. 30, 230 (1973).

[59] R. E. Troncoso and Á. S. Núñez, Brownian motion of massive skyrmions in magnetic thin films, Ann. Phys. 351, 850 (2014).

[60] C. Schütte, J. Iwasaki, A. Rosch, and N. Nagaosa, Inertia, diffusion, and dynamics of a driven skyrmion, Phys. Rev. B 90, 174434 (2014).

[61] S. Rohart, J. Miltat, and A. Thiaville, Path to collapse for an isolated Néel skyrmion, Phys. Rev. B 93, 214412 (2016).

[62] J. Miltat, S. Rohart, and A. Thiaville, Brownian motion of magnetic domain walls and skyrmions, and their diffusion constants, Phys. Rev. B 97, 214426 (2018).

[63] L. Rózsa, E. Simon, K. Palotás, L. Udvardi, and L. Szunyogh, Complex magnetic phase diagram and skyrmion lifetime in an ultrathin film from atomistic simulations, Phys. Rev. B 93, 024417 (2016).

[64] L. Zhao, Z. Wang, X. Zhang, J. Xia, K. Wu, H.-A. Zhou, Y. Dong, G. Yu, K. L. Wang, X. Liu, Y. Zhou, and W. Jiang, Spin-topology dependent brownian diffusion of skyrmions, arXiv:1901.08206v1.

[65] I. S. Lobanov, H. Jónsson, and V. M. Uzdin, Mechanism and activation energy of magnetic skyrmion annihilation obtained from minimum energy path calculations, Phys. Rev. B 94, 174418 (2016).

[66] D. Stosic, J. Mulkers, B. Van Waeyenberge, T. B. Ludermir, and M. V. Milošević, Paths to collapse for isolated skyrmions in few-monolayer ferromagnetic films, Phys. Rev. B 95, 214418 (2017).

[67] D. Cortés-Ortuño, W. Wang, M. Beg, R. A. Pepper, M.-A. Bisotti, R. Carey, M. Vousden, T. Kluyver, O. Hovorka, and H. Fangohr, Thermal stability and topological protection of skyrmions in nanotracks, Sci. Rep. 7, 4060 (2017).

[68] P. F. Bessarab, G. P. Müller, I. S. Lobanov, F. N. Rybakov, N. S. Kiselev, H. Jónsson, V. M. Uzdin, S. Blügel, L. Bergqvist, and A. Delin, Lifetime of racetrack skyrmions, Sci. Rep. 8, 3433 (2018).

[69] V. M. Uzdin, M. N. Potkina, I. S. Lobanov, P. F. Bessarab, and H. Jónsson, The effect of confinement and defects on the thermal stability of skyrmions, Physica B: Condensed Matter 549, 6 (2018).

[70] V. M. Uzdin, M. N. Potkina, I. S. Lobanov, P. F. Bessarab, and H. Jónsson, Energy surface and lifetime of magnetic skyrmions, J. Magn. Magn. Mater. 459, 236 (2018). 